\title{
SUBGROUPS AND AUTOMORPHISMS OF EXTENDED SCHOTTKY TYPE GROUPS $\left.{ }^{1}\right)$
}

\author{
BY \\ VICKI CHUCKROW
}

The purpose of this paper is to generalize some results on Schottky groups to extended Schottky type groups. Such groups represent compact Riemann surfaces with an even number of punctures.

The paper contains two results. In $\$ 3$ we prove that all finitely generated subgroups of extended Schottky type groups are also extended Schottky type groups. This is a direct consequence of a theorem of Maskit's.

Since an extended Schottky type $G$ is marked with a set of standard generators, the question arises which generators are standard for $G$. In $\$ 4$ we prove that under an automorphism of $G$ which preserves parabolic elements, standard generators map into standard generators. The proof relies on a theorem of automorphisms of free product groups of R. Prisco.

1. Definitions and notation. A Riemann surface $S$ is said to be a finite surface with $t$ punctures if it can be obtained from a compact Riemann surface by the removal of $t$ points. A retrosection on a Riemann surface is an oriented simple closed curve. If $\alpha$ is a retrosection on the Riemann surface then a retrosection $\beta$ is said to be conjugate to $\alpha$ if $\beta$ intersects $\alpha$ in only one point and $\alpha \cdot \beta=1$, i.e., the intersection number of $\alpha$ and $\beta$ is one. We then say that $(\alpha, \beta)$ is a retrosection pair. A system of retrosections of type $(r, s)$ on a finite Riemann surface $S$ of genus $r+s$ with $t$ punctures is a set of $r$ retrosections $\gamma_{1}, \ldots, \gamma_{r}$ and $s$ retrosection pairs $\lambda_{1}, \ldots, \lambda_{s}$ such that $S-\left\{\gamma_{1}, \ldots, \gamma_{r}, \lambda_{1}, \ldots, \lambda_{s}\right\}$ is homeomorphic to the $2 r+s+t$ times punctured sphere.

Let $G$ be a group of Möbius transformations viewed as automorphisms of the Riemann sphere $P$ and $\Lambda(G)$ the set of limit points of $G$, i.e., $\Lambda(G)$ is the union of the derived sets of $\left\{G z_{0}\right\}$ as $z_{0}$ ranges over $P$. We assume that $G$ is discontinuous, i.e., $\Lambda(G) \neq P$. Then the factor space $S_{G}=(P-\Lambda(G)) / G$ is a union of Riemann surfaces; the conformal structure of $S_{G}$ is defined by requring that the natural map $\pi$ of $P-\Lambda(G)$ onto $S_{G}$ be holomorphic. A connected region (an open set together with part of its boundary) $D \subset P-\Lambda(G)$ is said to be a fundamental region for the discontinuous group $G$ if $\bigcup_{g \in G} g(D)=P-\Lambda(G)$ and no two points of $D$ are equivalent under $G$.

Received by the editors November 6, 1967 and, in revised form, October 24, 1969.

(1) This research was supported in part by an NSF fellowship and in part by NSF Grant No. GP-6302.

Copyright (C) 1970, American Mathematical Society 
A group $G$ of Möbius transformations is said to be a group of extended Schottky type $(r, s, p)$ (or an E.S.T. group of type $(r, s, p)$ ) with standard generators $T_{1}, \ldots$, $T_{r}, W_{1}, \ldots, W_{p}, U_{1}, V_{1}, \ldots, U_{s}, V_{s}$, and defining curves $\Gamma_{1}, \Gamma_{1}^{\prime}, \ldots, \Gamma_{r}, \Gamma_{r}^{\prime}, \Delta_{1}, \Delta_{1}^{\prime}$, $\ldots, \Delta_{p}, \Delta_{p}^{\prime}, \Lambda_{1}, \ldots, \Lambda_{s}$ where $\Lambda_{i}$ is a topological rectangle with sides $\Omega_{i}, \Sigma_{i}, \Omega_{i}^{\prime}$, $\Sigma_{i}^{\prime}, i=1, \ldots, s$, if

(i) The curves are disjoint Jordan curves, except $\Delta_{k}, \Delta_{k}^{\prime}$ have a common point $p_{k}$, which bound a $2 r+p+s$ times connected domain $D$ such that $T_{j}(D) \cap D$ $=W_{k}(D) \cap D=U_{i}(D) \cap D=V_{i}(D) \cap D=\varnothing$.

(ii) $T_{j}\left(\Gamma_{j}\right)=\Gamma_{j}^{\prime}, W_{k}\left(\Delta_{k}\right)=\Delta_{k}^{\prime}, U_{i}\left(\Omega_{i}\right)=\Omega_{i}^{\prime}, V_{i}\left(\Sigma_{i}\right)=\Sigma_{i}^{\prime}, j=1, \ldots, r ; k=1, \ldots, p$; $i=1, \ldots, s$. (See Figure 1.)

(iii) $U_{i}$ and $V_{i}$ are commuting parabolic transformations.

(iv) Group $\left\{U_{i}, V_{i}\right\}$ is discontinuous.

(v) $W_{k}$ is parabolic with fixed point $p_{k}$.

We call $D \cup\left(\bigcup_{i=1}^{r} \Gamma_{i}\right) \cup\left(\bigcup_{k=1}^{p} \Delta_{k}\right) \cup\left(\bigcup_{j=1}^{s} \Omega_{j} \cup \Sigma_{j}\right)$ a standard fundamental region for $G$. Then $P-\Lambda(G)$ is connected and $(P-\Lambda(G)) / G$ is a finite Riemann surface $S_{G}$ of genus $r+s$ with $2 p$ punctures, with a set of $r+s$ disjoint curves $\gamma_{1}, \ldots, \gamma_{r}$, $\lambda_{1}, \ldots, \lambda_{s}$ distinguished on $S_{G}$ such that $\gamma_{1}, \ldots, \gamma_{r}, \lambda_{1}, \ldots, \lambda_{s}$ is a complete system of retrosections of type $(r, s)$. If $p=0$ then $G$ is said to be of Schottky type $(r, s)$ and $S_{G}$ is a compact surface.

Conversely, the fact that any finite Riemann surface of genus $r+s$ with $2 p$ punctures and a complete system of retrosections of type $(r, s)$ can be represented
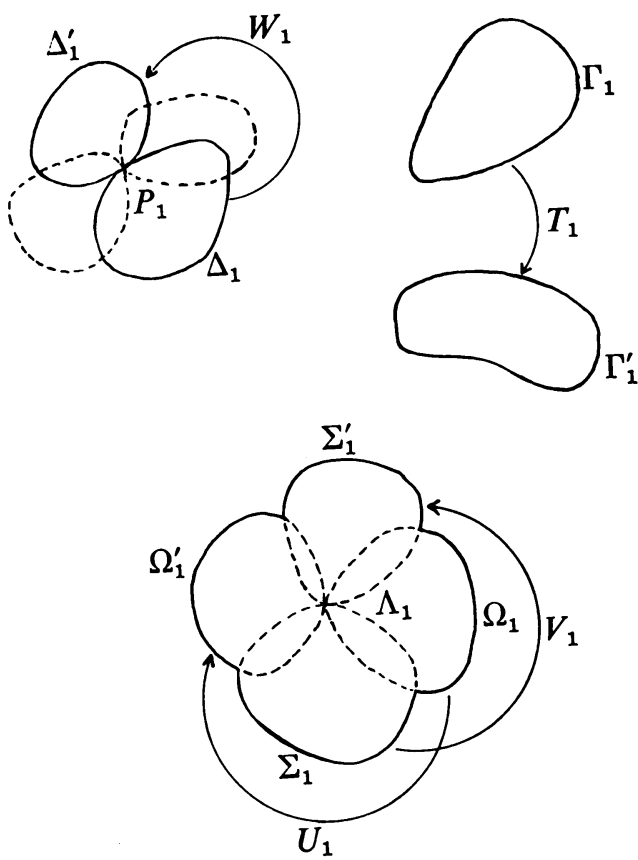

FIGURE 1. E.S.T. group of type $(1,1,1)$ 
by an E.S.T. group of type $(r, s, p)$ is a classical theorem and can be proved by Bers' continuity method [3].

\section{Properties of E.S.T. groups.}

Property 1. Every E.S.T. group $G$ of type $(r, s, p)$ can be extended to a Schottky type group of type $(r, s+p)$.

Proof. Draw curves $\chi_{k}, \chi_{k}^{\prime}$ through $p_{k}$ such that $\Delta_{k}, \chi_{k}, \Delta_{k}^{\prime}, \chi_{k}^{\prime}$ "form" a topological rectangle. Let $X_{k}$ be a parabolic transformation with fixed point $p_{k}$ identifying $\chi_{k}$ and $\chi_{k}^{\prime}$ such that the group $\left\{X_{k}, W_{k}\right\}$ is discontinuous. Then $G$ is a subgroup of the group $H$ generated by $G$ and the $X_{k}, k=1, \ldots, p$, where $H$ is of Schottky type $(r, s+p)$ (see Figure 1$)$.

PROPERTY 2. If $G$ is an E.S.T. group with standard generators $T_{1}, \ldots, T_{r}$, $W_{1}, \ldots, W_{p}, U_{1}, V_{1}, \ldots, U_{s}, V_{s}$, then $G$ has the presentation $\left\langle T_{1}, \ldots, T_{r}, W_{1}, \ldots\right.$, $\left.W_{p}, U_{1}, V_{1}, \ldots, U_{s}, V_{s} ;\left[U_{j}, V_{j}\right]=1, j=1, \ldots, s\right\rangle$, i.e., $G$ is the free product of $r+p$ infinite cyclic groups and $s$ free abelian groups on two generators.

Proof. The proof follows directly from Klein's Combination Theorem which states that in the formation of groups by combination no new relations are introduced [2, p. 209].

Property 3. If $G$ is an E.S.T. group then $G$ has no elliptic elements.

Proof. Any elliptic element belonging to $G$ would have to be of finite order as $G$ is discontinuous. But this is impossible as this would introduce a new relation in the combination group.

ProperTy 4. An element belonging to an E.S.T. group $G$ is parabolic iff it is conjugate in $G$ to an element of the form $W_{k}^{n}$ or $U_{j}^{n} V_{j}^{m}$ for some integers $n$ and $m$.

We first prove a lemma. If $\Gamma$ is a closed curve then Int $\Gamma$ denotes the interior of $\Gamma$ and $\mathrm{Cl}$ (Int $\Gamma$ ) the closure of Int $\Gamma$.

LEMMA 1. Let $G$ be an E.S.T. group with standard generators $T_{1}, \ldots, T_{r}, W_{1}, \ldots$, $W_{p}, U_{1}, V_{1}, \ldots, U_{s}, V_{s}$, and defining curves $\Gamma_{1}, \Gamma_{1}^{\prime}, \ldots, \Gamma_{s}, \Gamma_{s}^{\prime}, \Delta_{1}, \Delta_{1}^{\prime}, \ldots, \Delta_{p}$, $\Delta_{p}^{\prime}, \Lambda_{1}, \ldots, \Lambda_{s}$ bounding a domain $D$. Then if $W \in G$ and

(i) $W=T_{i}^{\alpha}(\cdots)$, then

(a) $W(D) \subset$ Int $\Gamma_{\mathfrak{i}}^{\prime}$ and $W$ has a fixed point in $\mathrm{Cl}$ (Int $\left.\Gamma_{i}^{\prime}\right)$ if $\alpha>0$.

(b) $W(D) \subset$ Int $\Gamma_{i}$ and $W$ has a fixed point in $\mathrm{Cl}\left(\right.$ Int $\left.\Gamma_{i}\right)$ if $\alpha<0$.

(ii) $W=W_{k}^{\alpha}(\cdots)$, then

(a) $W(D) \subset$ Int $\Delta_{k}^{\prime}$ and $W$ has a fixed point in Cl (Int $\Delta_{k}^{\prime}$ ) if $\alpha>0$.

(b) $W(D) \subset$ Int $\Delta_{k}$ and $W$ has a fixed point in $\mathrm{Cl}$ (Int $\Delta_{k}$ ) if $\alpha<0$.

(iii) $W=U_{j}^{\alpha} V_{j}^{\beta}(\cdots),|\alpha|+|\beta|>0$, then $W(D) \subset$ Int $\Lambda_{j}$ and $W$ has a fixed point in $\mathrm{Cl}$ (Int $\Lambda_{j}$ ), where we assume $W$ is a freely reduced word in the generators of $G$.

Proof. We prove only case (iii). The first half of the conclusion is just a special case of Klein's Combination Theorem. Now, using the fact that $W(D) \subset$ Int $\Lambda_{j}$, it is not difficult to check that $W\left(\operatorname{Int} \Lambda_{j}\right) \subset \operatorname{Int} \Lambda_{j}$ and thus conclude that $W$ has a fixed point in $\mathrm{Cl}$ (Int $\left.\Lambda_{j}\right)$. 
Proof of Property 4. Clearly all conjugates of $U_{j}^{n} V_{j}^{m}$ and $W_{k}^{n}$ are parabolic. On the other hand if $A \in G$ is not conjugate to an element of this form, then without loss of generality we may assume $A$ is not of this form. For example as a reduced word in the generators of $G, A$ might be of the form $Z_{1} T_{i}^{-1} U_{j}^{n} V_{j}^{m} Z_{2}$. Then a conjugate of $A, A^{\prime}=U_{j}^{n} V_{j}^{m} Z_{2} Z_{1} T_{i}^{-1}$. By Lemma $1, A^{\prime}$ has a fixed point in $\mathrm{Cl}$ (Int $\Lambda_{j}$ ) and $A^{\prime-1}$ and hence $A^{\prime}$ has a fixed point in $\mathrm{Cl}$ (Int $\left.\Gamma_{i}^{\prime}\right)$. Thus $A$ is loxodromic. The proof of all other cases is similar.

Property 4 is a special case of a more general theorem which we state as follows:

THEOREM 1. In a combination group $G$ satisfying the hypothesis of Klein's Combination Theorem an element is parabolic if and only if it is conjugate in $G$ to a parabolic element belonging to one of the original groups.

The proof of this theorem is essentially the same as that of Property 4.

3. Subgroups of E.S.T. groups. We say a domain $D \subset P$ is of span zero if the only univalent analytic functions defined on $D$ are Möbius transformations. It is classical that if $G$ is an E.S.T. group then $P-\Lambda(G)$ is of span zero (see Property 1 and [8]). On the other hand Maskit has proven that the property of $P-\Lambda(G)$ being of span zero essentially characterizes E.S.T. groups. Namely we have the following proposition:

Proposition 2. A discontinuous group is an E.S.T. group if and only if $G$ is finitely generated, $G$ has no elliptic elements and $P-\Lambda(G)$ is of span zero.

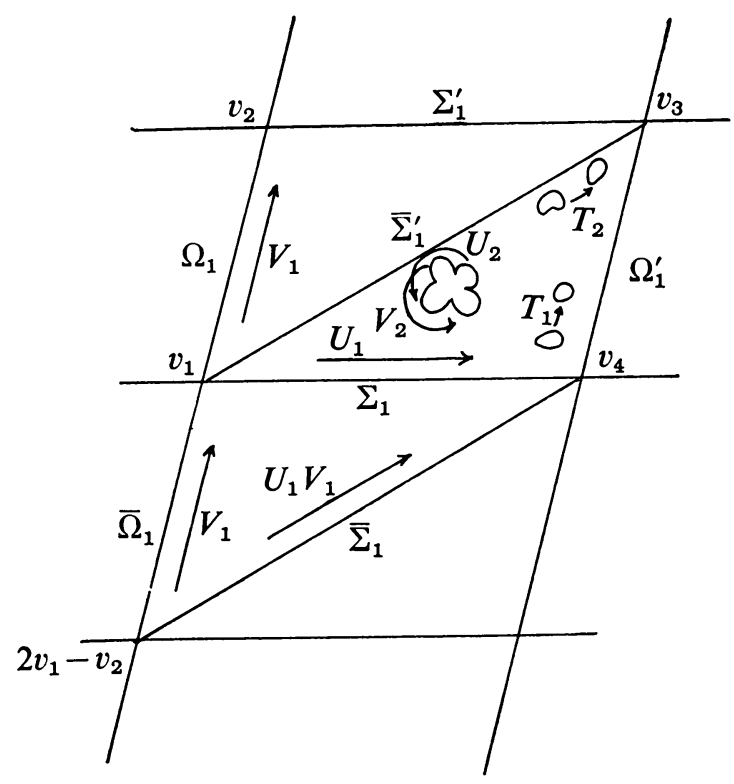

FIGURE 2. Automorphism of type $\mathrm{E}_{3}(\mathrm{c})$ 
Outline of proof $\left({ }^{2}\right)$. The only if part is clear. Conversely since $\Lambda(G)$ has span zero, $\Lambda(G)$ is connected, i.e., $G$ has exactly one component $\Delta=\Lambda(G)$, and $\Delta$ is invariant under $G$. Let $S=\Delta / G$. Since $G$ is finitely generated Ahlfors' Theorem [1] implies $S$ is a finite surface. By the Planarity Theorem [6] the covering of $S$ by $\Delta$ is given by a set of simple disjoint loops on $S$ ( $G$ has no elements of finite order).

Starting with $S$ and the set of loops construct (see [5]) a new group $G^{*}$ with invariant component $\Delta^{*}$ which gives the same uniformization of $S$. By conformal invariance $\Delta^{*}$ also has span zero.

$G^{*}$ is constructed as follows: Cut $S$ along each of the loops and fill in the holes with discs. This gives a collection of finite surfaces $S_{1}, \ldots, S_{k}$. For each $i=1, \ldots, k$ let $G_{i}$ be the Fuchsian or elementary group representing $S_{i}$. Form the free product (Klein's Combination Theorem) of the $G_{i}$ and the resulting group is a quasiconformal deformation of $G$ [5]. Since $\Delta^{*}$ has span zero, each $G_{i}$ must be an elementary group. Thus $G^{*}$ is formed via Klein's Combination Theorem as the free product of elementary groups without elliptic elements and we conclude that $G$ is an E.S.T. group.

THEOREM 2. Every finitely generated subgroup $H$ of an E.S.T. group $G$ is an E.S.T. group.

Proof. Since $H$ is a subgroup of $G, \Lambda(H) \subset \Lambda(G)$ and thus $P-\Lambda(H) \supset P-\Lambda(G)$ from which it follows that $P-\Lambda(H)$ is of span zero. The theorem now follows from Proposition 2.

4. Automorphisms of E.S.T. groups. We know that if $G$ is an E.S.T. group of type $(r, s, p)$ then there exists a standard set of generators for $G$ such that

$$
G=\left\langle T_{1}, \ldots, T_{r}, W_{1}, \ldots, W_{p}, U_{1}, V_{1}, \ldots, U_{s}, V_{s} ;\left[U_{j}, V_{j}\right]=1, j=1, \ldots, s\right\rangle .
$$

Under an automorphism of $G$ which preserves the type of parabolic elements the standard generators go into other generators, $T_{i} \rightarrow T_{i}^{\prime}, W_{k} \rightarrow W_{k}^{\prime}, U_{j} \rightarrow U_{j}^{\prime}$, $V_{j} \rightarrow V_{j}^{\prime}$ such that in terms of these new generators $G$ has the presentation

$$
\left\langle T_{1}^{\prime}, \ldots, T_{r}^{\prime}, W_{1}^{\prime}, \ldots, W_{k}^{\prime}, U_{1}^{\prime}, V_{1}^{\prime}, \ldots, U_{s}^{\prime}, V_{s}^{\prime} ;\left[U_{j}^{\prime}, V_{j}^{\prime}\right]=1, j=1, \ldots, s\right\rangle .
$$

We prove in this section that the generators $T_{i}^{\prime}, W_{k}^{\prime}, U_{j}^{\prime}, V_{j}^{\prime}$ are also standard for $G$. To this end we examine the automorphisms of $G$.

Automorphism THEOREM. If an E.S.T. group

$$
G=\left\langle T_{1}, \ldots, T_{r}, W_{1}, \ldots, W_{k}, U_{1}, V_{1}, \ldots, U_{s}, V_{s} ;\left[U_{j}, V_{j}\right]=1, j=1, \ldots, s\right\rangle
$$

then every automorphism of $G$ which preserves the type of parabolic elements can be obtained by finite repeated application of the following elementary automorphism:

$\left(^{2}\right)$ The proof was communicated to me by B. Maskit. 


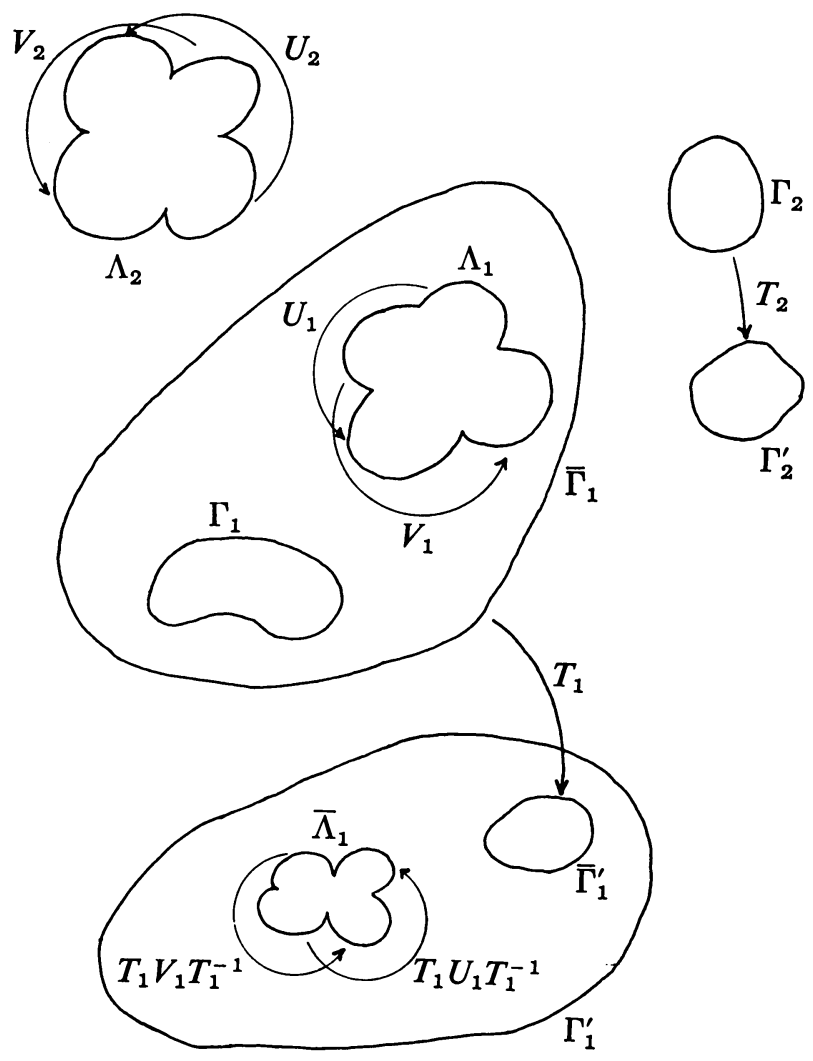

FIGURE 3. Automorphism of type $E_{4}(a)$

$\mathrm{E}_{1}$ (a) $T_{j} \rightarrow T_{i}, T_{i} \rightarrow T_{j}, i \neq j$ (by this we mean map $T_{i}$ into $T_{j}, T_{j}$ into $T_{i}$ and leave all other generators fixed); (b) $T_{i} \rightarrow T_{i}^{-1}$; (c) $T_{i} \rightarrow T_{k} T_{i}, i \neq k$.

$\mathrm{E}_{2}$ (a) $W_{j} \rightarrow W_{i}, W_{i} \rightarrow W_{j}, i \neq j$; (b) $W_{i} \rightarrow W_{i}^{-1}$; (c) $W_{i} \rightarrow W_{k} W_{i}, i \neq k$.

$\mathrm{E}_{3}$ (a) $U_{j} \rightarrow V_{j}, V_{j} \rightarrow U_{j}$; (b) $U_{j} \rightarrow U_{j}^{-1}$; (c) $U_{i} \rightarrow U_{i} V_{i}$.

$\mathrm{E}_{4}$ (a) $U_{j} \rightarrow T_{i} U_{j} T_{i}^{-1}, \quad V_{j} \rightarrow T_{i} V_{j} T_{i}^{-1}$; (b) $U_{j} \rightarrow W_{i} U_{j} W_{i}^{-1}, V_{j} \rightarrow W_{i} V_{j} W_{i}^{-1}$; (c) $U_{j} \rightarrow V_{i} U_{j} V_{i}^{-1}, V_{j} \rightarrow V_{i} V_{j} V_{i}^{-1}, i \neq j$.

$\mathrm{E}_{5}$ (a) $T_{i} \rightarrow V_{j} T_{i}$; (b) $T_{i} \rightarrow W_{j} T_{i}$.

$\mathrm{E}_{6} U_{j} \rightarrow U_{k}, U_{k} \rightarrow U_{j}, V_{j} \rightarrow V_{k}, V_{k} \rightarrow V_{j}, j \neq k$.

For a proof of this theorem see [7].

We show that under the elementary automorphisms $E_{1}-E_{6}$ standard generators for $G$ go into standard generators. Certain cases, namely $\mathrm{E}_{1}(\mathrm{a}), \mathrm{E}_{1}(\mathrm{~b}), \mathrm{E}_{2}(\mathrm{a})$, $\mathrm{E}_{2}(\mathrm{~b}), \mathrm{E}_{3}(\mathrm{a}), \mathrm{E}_{3}(\mathrm{~b}), \mathrm{E}_{6}$ are trivial. For the following lemmas we assume that $G$ is an E.S.T. group with presentation as in the preceding theorem, and that those generators are standard for $G$. 
LEMMA 2. Automorphisms of type $\mathrm{E}_{1}(\mathrm{c})$ preserve the standard generators of $G$.

Proof. The proof is an exact repetition of that of Lemma 1 of [4].

Lemma 3. Automorphisms of type $\mathrm{E}_{3}(\mathrm{c})$ preserve the standard generators of $G$.

Proof. We assume $i=1$ and that the common fixed point $p$ of $U_{1}, V_{1}$ is $\infty$. Let the vertices of the topological rectangle $\Lambda_{1}=\Omega_{1} \Sigma_{1} \Omega_{1}^{\prime} \Sigma_{1}^{\prime}$ be $v_{1}, v_{2}, v_{3}, v_{4}$ so that $U_{1}\left(v_{1}\right)=v_{4}, \quad U_{1}\left(v_{2}\right)=v_{3}, \quad V_{1}\left(v_{1}\right)=v_{2}$, and $V_{1}\left(v_{4}\right)=v_{3}$. We draw a "diagonal" $\bar{\Sigma}_{1}^{\prime}$ connecting $v_{1}$ and $v_{3}$ in such a way that $\bar{\Sigma}_{1}^{\prime} \subset$ Int $\Lambda_{1}$ and the topological triangle $\Omega_{1}^{\prime} \Sigma_{1} \bar{\Sigma}_{1}^{\prime}$ contains in its interior all the other defining curves of $G$ (see Figure 2). Let $\bar{\Sigma}_{1}=V_{1}^{-1}\left(\bar{\Sigma}_{1}^{\prime}\right), \bar{\Omega}_{1}=V_{1}^{-1}\left(\Omega_{1}\right)$ and $\bar{\Lambda}_{1}=\bar{\Omega}_{1} \bar{\Sigma}_{1} \Omega_{1}^{\prime} \bar{\Sigma}_{1}^{\prime}$. It is then easily verified that the generators $T_{1}, \ldots, T_{r}, W_{1}, \ldots, W_{k}, U_{1} V_{1}, V_{1}, U_{2}, V_{2}, \ldots, U_{s}, V_{s}$, are standard generators for $G$ with defining curves $\Gamma_{1}, \Gamma_{1}^{\prime}, \ldots, \Gamma_{r}, \Gamma_{r}^{\prime}, \Delta_{1}, \Delta_{1}^{\prime}, \ldots, \Delta_{k}, \Delta_{k}^{\prime}$, $\bar{\Lambda}_{1}, \Lambda_{2}, \ldots, \Lambda_{s}$.

Lemma 4. Automorphisms of type $\mathrm{E}_{4}(\mathrm{a})$ preserve the standard generators of $G$.

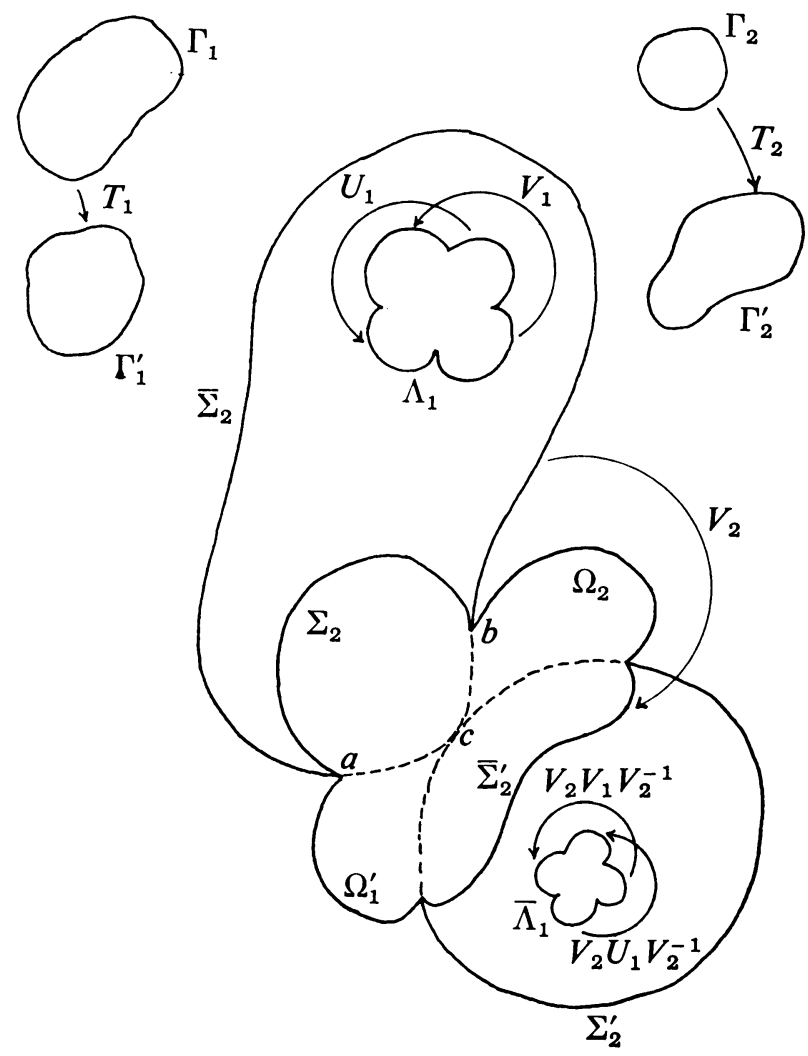

Figure 4. Automorphism of type $\mathrm{E}_{4}(\mathrm{c})$ 
Proof. Figure 3 suffices to indicate the outline of the proof. Here $i=j=1$. The details of the proof are omitted as they are similar to the previous argument.

LEMMA 5. Automorphisms of type $\mathrm{E}_{4}(\mathrm{c})$ preserve the standard generators of $G$.

Proof. See Figure 4. Here $i=2, j=1$.

LEMMA 6. Automorphisms of type $\mathrm{E}_{5}(\mathrm{a})$ preserve the standard generators of $G$.

Proof. See Figure 5. Here $i=1, j=2$.

We omit the lemmas for automorphisms of type $E_{2}(c), E_{4}(b)$, and $E_{5}(b)$ as they are similar to the preceding ones.

THEOREM 3. If $G$ is an E.S.T. group then under any automorphism of $G$ preserving parabolic elements, standard generators are mapped onto standard generators.

Proof. The proof follows directly from the preceding lemmas and the automorphism theorem.

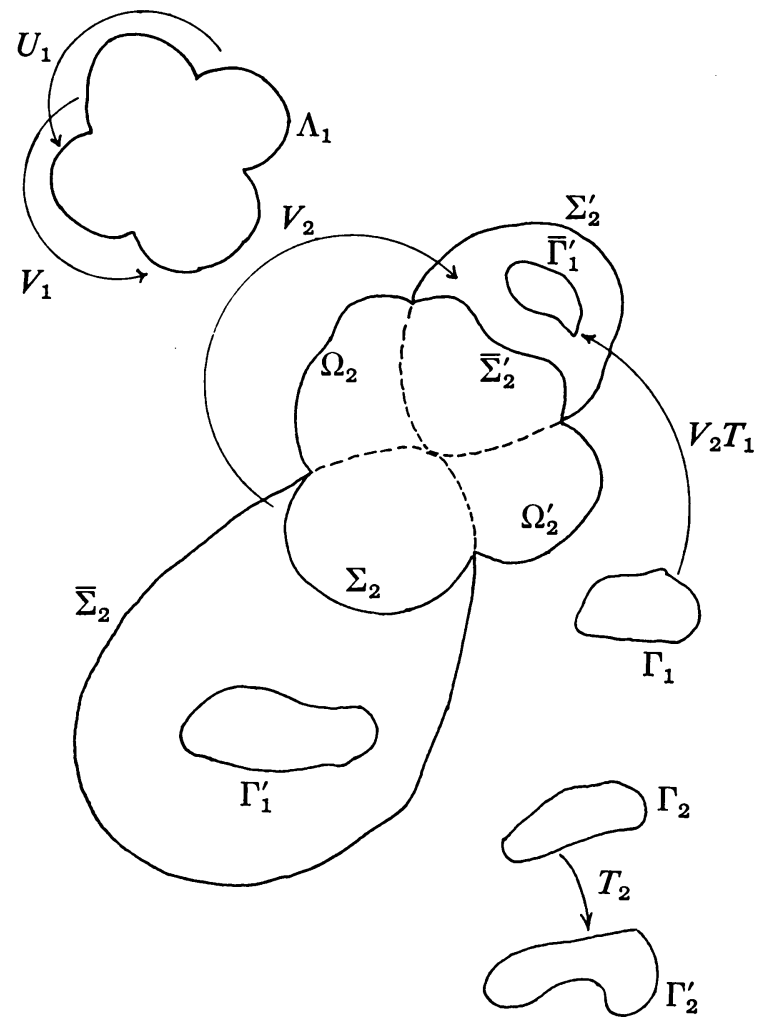

Figure 5. Automorphism of type $\mathrm{E}_{5}(\mathrm{a})$ 


\section{BIBLIOGRAPHY}

1. L. V. Ahlfors, Finitely generated Kleinian groups, Amer. J. Math. 86 (1964), 413-429. MR 29 \#4890.

2. P. Appell and E. Goursat, Théorie des fonctions algébriques d'une variable. Vol. II: Fonctions automorphes, Gauthier-Villars, Paris, 1930.

3. L. Bers, Uniformization by Beltrami equations, Comm. Pure Appl. Math. 14 (1961), 215-228. MR 24 \#A2022.

4. V. Chuckrow, On Schottky groups with applications to kleinian groups, Ann of Math. (2) 88 (1968), 47-61. MR 37 \#2987.

5. B. Maskit, Construction of Kleinian groups, Proc. Conf. Complex Analysis (Minneapolis, 1964), Springer-Verlag, Berlin and New York, 1965, pp. 281-296. MR 31 \#2396.

6. - A theorem on planar covering surfaces with applications to 3-manifolds, Ann. of Math. (2) 81 (1965), 341-355. MR 30 \#2472.

7. R. Prisco, On free products, conjugating factors, and Hopfian groups, Thesis, Adelphi University, Garden City, N. Y., 1967.

8. L. Sario, Über Riemannsche Flächen mit hebbarem Rand, Ann. Acad. Sci. Fenn. Ser. A.I. Math.-Phys. No. 50 (1948). MR 10, 365.

The City University of New York, City College, NeW YoRK, New YoRK 10031 